\title{
Informe de caso y revisión bibliográfica: Tetralogía de Fallot
}

\author{
Case report and bibliographic review: Tetralogy of Fallot \\ López Rodríguez Javier Arturo', Carchi Heras Mayra Alexandra'
}

VOLUMEN 37 | No 2 I SEPTIEMBRE 2019

FECHA DE RECEPCIÓN: $\quad 30 / 04 / 2019$ FECHA DE APROBACIÓN: 08/08/2019 FECHA PUBLICACIÓN: 10/09/2019

\section{Clínica Santa Inés}

\begin{tabular}{r|l} 
Caso & Clinical \\
Clínico & Case
\end{tabular}

DOI: https://doi.org/10.18537/RFCM.37.02.07

Correspondencia:

javier.lopezQucuenca.edu.ec

Dirección:

Santiago Carrasco 4-128 y Cornelio Merchán

Código Postal:

010204

Celular:

0984994206

Cuenca - Ecuador

\section{RESUMEN}

La Tetralogía de Fallot es la enfermedad cardiaca congénita cianótica más común y requiere una corrección quirúrgica temprana; se presenta el caso de una paciente que a los seis meses es sometida a cirugía paliativa de derivación sistémico pulmonar con fistula Blalock Taussing modificada con injerto de Goretex, su cuadro no mejora con el paso de los años debiéndose realizar cirugía de corrección total. Al ingreso presenta disnea de medianos esfuerzos, astenia, cianosis peribucal y distal que ceden con el reposo; en tórax presencia de cicatriz infra axilar compatible con cirugía paliativa previa, corazón: R1 y R2 rítmicos, normo fonéticos, presencia de soplo holosistólico en foco pulmonar y soplo infraclavicular izquierdo de fístula sistémico pulmonar. En la radiografía de tórax se observa aumento de índice cardiotorácico e hilios vasculares, el ecocardiograma pre quirúrgico transtorácico reporta estenosis infundibular por hipertrofia severa del miocardio, gradiente máximo de $90 \mathrm{~mm} / \mathrm{Hg}$. La cirugía de corrección total de Tetralogía de Fallot luego de procedimientos paliativos previos, con control por ecocardiograma transesofágico transoperatorio mejora la calidad de vida del paciente.

Palabras clave: cardiopatías congénitas, Tetralogía de Fallot, discusiones bioéticas, procedimientos quirúrgicos operativos.

\section{ABSTRACT}

Tetralogy of Fallot is the most common cyanotic congenital heart disease and requires early surgical correction, this is a case of a patient who at six months undergoes palliative pulmonary systemic bypass surgery with modified Blalock Taussing fistula with Goretex graft, his condition does not improve with the passing of the years and a total correction surgery must be performed.

Upon admission, the patient presents: dyspnea of medium efforts, asthenia, peribucal and distal cyanosis that yield with rest; in thorax presence of infra axillary scar compatible with previous palliative surgery, heart: rhythmic R1 and R2, phonetic norm, presence of holosystolic murmur in pulmonary focus and left infraclavicular murmur of pulmonary systemic fistula.

The chest radiograph shows an increase in cardiothoracic index and vascular congenital and prominent hilum, transthoracic pre-surgical echocardiography is performed where infundibular stenosis is observed due to severe myocardial hypertrophy, maximum gradient of $90 \mathrm{~mm} / \mathrm{Hg}$.

Tetralogy of Total Fall correction surgery after previous palliative procedures, with transoperative transesophageal echocardiogram control, improves the patient's quality of life. 
Key words: heart defects congenital, Tetralogy of Fallot, bioethical issues, surgical procedures.

\section{INTRODUCCION}

La tetralogía de Fallot representa entre 3.5 y $8 \%$ de los defectos cardiacos congénitos; conlleva un estado de hipoxia crónica [1]; no se conoce con precisión el origen de la anomalía embriológica que genera hipoplasia infundibular que luego provocará hipertrofia ventricular derecha secundaria al grado de obstrucción de la zona pulmonar, una desviación anterior e izquierda del septum infundibular, el cual se encuentra disociado de la trabécula septomarginal, la comunicación interventricular y de cierto grado de la dextro posición de la aorta [2]. Esta cardiopatía en ocasiones se asocia con comunicación interauricular en el $35 \%$, arco aórtico derecho $30 \%$, persistencia de vena cava izquierda $10 \%$, y otras malformaciones menos frecuentes [3].

Aunque la Tetralogía de Fallot puede presentarse como parte de un síndrome conocido, esta lesión típicamente ocurre esporádicamente sin otras anomalías. Aproximadamente el 15 por ciento de los pacientes con Tetralogía de Fallot presentan síndromes asociados como el Síndrome de Down (trisomía 21), Síndrome de Alagille (mutaciones en JAG1), Síndrome de Di George y síndromes velocardiofaciales (supresión en el cromosoma 22q11). Puede haber genes de susceptibilidad para Tetralogía de Fallot dentro de la última región del cromosoma 22q11 en niños sin anomalías extracardíacas, y el síndrome de deleción 22q11.2 no se reconoce en muchos pacientes adultos con Tetralogía de Fallot $[4,5]$

Las consecuencias fisiológicas de la tetralogía de Fallot dependen en gran medida del grado de obstrucción del flujo de salida del ventrículo derecho. La presión en el ventrículo derecho (VD) refleja la del ventrículo izquierdo (VI), como resultado, la dirección del flujo sanguíneo a través del VD estará determinada por la ruta de menor resistencia para el flujo sanguíneo. Si no existe resistencia a la salida del VD la sangre fluirá hacia los pulmones, pero si existe mayor resistencia por estenosis de la válvula pulmonar, la sangre fluirá más fácilmente al VI a través de la comunicación interventricular, por lo tanto, llegará a la circulación sistémica sin oxigenarse por lo que se presenta cianosis. La estenosis pulmonar es la responsable de la gravedad de la cardiopatía ya que de ella depende el aporte de sangre a los pulmones, cuando más severa es la obstrucción in- fundibular, mayor será el cortocircuito desde el ventrículo derecho a la aorta, y más importante será la cianosis y el grado de oligohemia pulmonar [6].

La hipoxia en crisis es frecuente, cerca de la tercera parte de pacientes que se intensifican gradualmente en relación con el grado de estenosis pulmonar [7].

Es la enfermedad cardíaca cianótica más común después del período neonatal, representando aproximadamente el $3,5 \%-10 \%$ de las cardiopatías congénitas, el $2 \%$ de 2,256 muertes neonatales atribuibles a defectos cardíacos congénitos se debió a la tetralogía de Fallot en Estados Unidos en 2003-2006, su prevalencia estimada en Estados unidos es 3.92 por 10,000 nacidos vivos, o 1,574 casos por años y es una de las lesiones cardíacas congénitas más comunes que requieren intervención en el primer año de vida. Ocurre de igual manera en hombres y mujeres [8].

\section{CASO CLINICO}

Paciente femenina de 5 años de edad, procedente de Girón, provincia del Azuay, producto de primera gestación, cuya madre cursa con hospitalización en el primer trimestre por diagnóstico de pielonefritis y amenaza de parto prematuro en tratamiento con antibioticoterapia y progesterona, siendo dada de alta luego de dos semanas sin complicaciones; posteriores controles y ecografías sin aparente patología. Nace por parto eutócico a término, no presenta llanto inmediato al nacer, sin embargo, no requirió ingreso a neonatología y sin complicaciones es dada de alta junto con madre.

A los 6 meses de edad, en junio de 2.014 presenta crisis de cianosis peri bucal tras el llanto y diaforesis durante la lactancia motivo por el que se realiza radiografía de tórax evidenciando corazón en forma de "zapato sueco", electrocardiograma mismo que es normal, y ecocardiograma mismo que informa de estenosis infundibular importante, comunicación interventricular de $5 \mathrm{~mm}$, cabalgamiento de la aorta de $50 \%$ y ramas pulmonares pequeñas, sin evidencia de persistencia de conducto arterioso, siendo diagnosticada de Tetralogía de Fallot, ante este resultado se realiza cirugía paliativa de derivación sistémico pulmonar, por lo que se realiza fistula Blalock Taussing modificada con injerto de Goretex de $5 \mathrm{~mm}$ (Figura № 1), esta intervención paliativa tuvo como objetivo mejorar la hipoxemia, ya que al aumentar el flujo a nivel pulmonar 
se facilita su saturación y además permite el crecimiento controlado de las ramas pulmonares para una posterior corrección total cuando nuevamente se manifiesten crisis de cianosis.

Luego de la cirugía permanece en unidad de cuidados intensivos pediátricos por 10 días, presentando en el transcurso hiperfunción de fistula sistémico pulmonar izquierda con hipercongestión unilateral pulmonar derecha sin observarse hiperflujo izquierdo, el mismo que es controlado; es referida a hospitalización general en buenas condiciones y 6 días después es dada de alta con tratamiento farmacológico a base de propanolol 10 mg y aspirina $100 \mathrm{mg}$ una vez al día.

\section{Figura $N^{0} 1$}

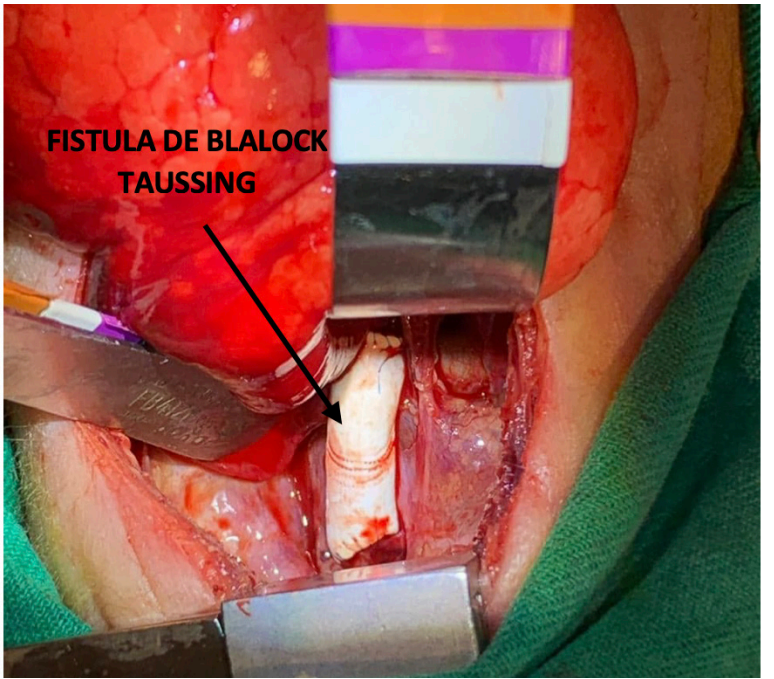

Fistula sistémico pulmonar de Blalock Taussing modificada con injerto de GORETEX de $5 \mathrm{~mm}$, primera cirugía paliativa realizada 17 de junio del 2014.

El seguimiento clínico de la paciente fue realizado de manera semestral, con controles continuos anuales de ecocardiografía, mismos que informan de fistula permeable y crecimiento de las ramas pulmonares de manera homogénea, en algunas ocasiones se ha evidenciado crecimiento no equilibrado de ramas pulmonares por falta de adecuada técnica quirúrgica, además llevó farmacoterapia a base de clopidogrel para evitar obstrucción de la fistula, y propanolol para control de frecuencia cardiaca evitando episodios de disnea y cianosis por aumento de contractibilidad del ventrículo derecho, misma que se mantuvo durante estos años modificando únicamente dosis de acuerdo a peso de la paciente, entre los cuidados no farmacológicos se pidió que paciente evite actividad física de alta intensidad y ante signos de alarma acudir a nuevo control.

En presente año se evidencia disminución de la saturación de oxígeno basal de la paciente, que anteriormente se mantenía sobre $80 \%$, y actualmente desciende a $70 \%$ debido a mayores requerimientos basales por crecimiento de la paciente y disminución de flujo a través de fistula debido a que esta se vuelve insuficiente para la edad y desarrollo actual de la paciente, afectando su calidad de vida, motivo por el que se decide intervención para corrección total de Tetralogía de Fallot.

La paciente al ingreso, con 5 años 4 meses de edad presentó disnea de medianos esfuerzos y astenia, además de cianosis peri bucal y distal que ceden con el reposo. Al examen físico: peso $32 \mathrm{~kg}$, talla $115 \mathrm{~cm}$, saturación de oxigeno $70 \%$, índice de masa corporal 24, apariencia general buena, actividad psicomotriz conservada; en boca, cianosis bucal, múltiples caries dentales y en tórax presencia de cicatriz infra axilar compatible con cirugía paliativa previa, corazón: R1 y R2 rítmicos, normo fonéticos, presencia de soplo holosistólico en foco pulmonar y soplo infraclavicular izquierdo de fístula sistémico pulmonar.

En la radiografía de tórax se observa aumento de índice cardiotorácico e hilios vasculares de aspecto congestivo y prominente (Figura $\mathrm{N}^{\circ} 2$ ). Se realiza electrocardiograma mismo que reporta ritmo sinusal, sin alteraciones significativas.

Figura $\mathrm{N}^{0} 2$.

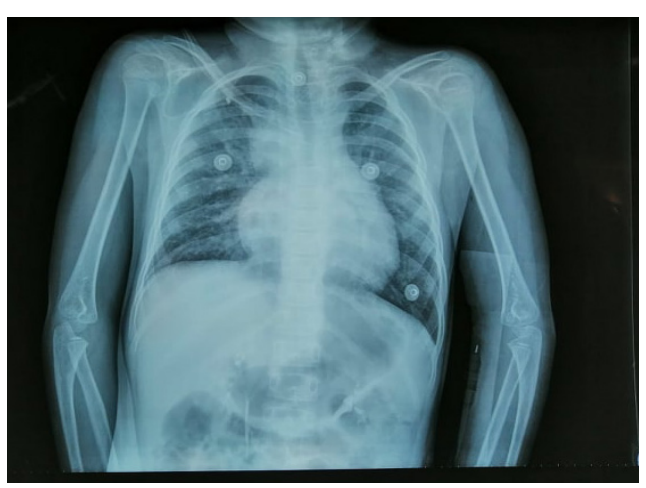

Radiografía de Tórax, paciente con tetralogía de Fallot 5 años después de realización fistula sistémico pulmonar de Blalock Taussing, donde se observa cardiomegalia. 
Se realiza ecocardiograma pre quirúrgico transtorácico donde se observa estenosis infundibular por hipertrofia severa del miocardio, gradiente máximo de $90 \mathrm{~mm} / \mathrm{Hg}$, desvío del tabique hacia la derecha, además válvula pulmonar engrosada y estenótica con buen diámetro de ramas de arterias pulmonares, fistula sistémico pulmonar izquierda con buen flujo aparentemente de $4 \mathrm{~mm}$ de diámetro y relación inferior en tamaño a ramas pulmonares, rama pulmonar y derecha de buen diámetro $8 \mathrm{~mm}$ rama derecha, $9 \mathrm{~mm}$ rama izquierda aproximadamente, comunicación interventricular de $10 \mathrm{~mm}$ y cabalgamiento de la aorta de $50 \%$.

Antes de la cirugía se realiza extracción de piezas dentales en mal estado, debido a que en la cirugía al utilizar circulación extracorpórea existe riesgo de diseminación de gérmenes que pueden provocar endocarditis al colonizar el parche pericárdico, mismo que es susceptible de infección en los primeros meses.

Mediante cateterismo cardiaco se evidencia ventrículo derecho hipertrófico con infundíbulo estrecho, ramas pulmonares de buen tamaño, ventrículo izquierdo sin alteración, aorta cabalgada, comunicación interventricular de infundíbulo $6 \mathrm{~mm}$ con flujo bidireccional, arco aórtico derecho, se observa paso de flujo desde la arteria subclavia izquierda hacia la rama pulmonar izquierda a través de fistula sistémico pulmonar. (Figura $N^{\circ} 3$ )

\section{Figura No 3}

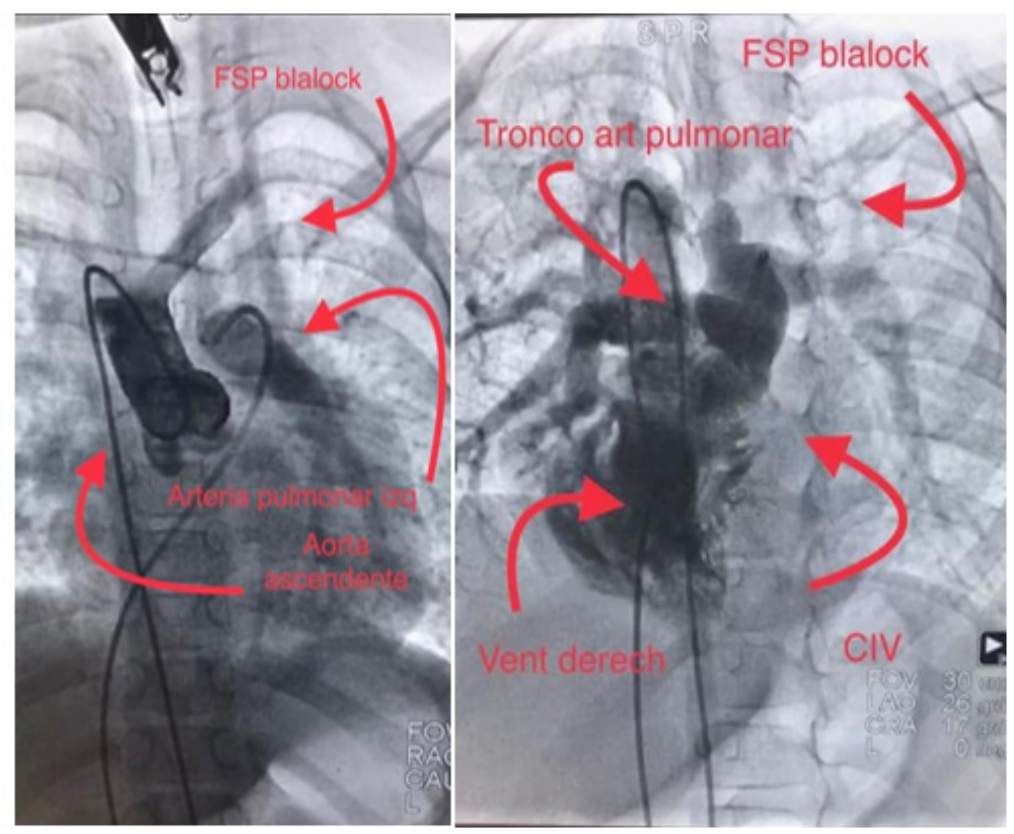

Fistula de Blalock Taussing funcionante, de tamaño insuficiente para el flujo sanguíneo recibido a la edad actual de la paciente.

En el estudio previo se observa una fistula sitemico pulmonar funcionante, sin embargo insuficiente para la edad de la paciente por lo que se decidió correccion total de Tetralogia de Fallot, este procedimiento quirúrgico consistió en una cirugía de corazón abierto por esternotomia total, con disección anterior de la fistula sección y sutura de la misma para evitar hiperflujo a nivel pulmonar, ampliacion del tracto de salida del ventriculo derecho a través de resección de zona fibrótica de la región infundibular, ampliacion de la arteria pulmonar con parche de pericardio bovino y cierre de la comunicación interventricular; misma que se realiza sin complicaciones, es ingresada a unidad de cuidados intensivos pediátricos bajo ventilación mecánica, tratamiento clínico y quirúrgico. 
Durante el acto operatorio se cumple control por ecocardiograma transesofágico transoperatorio donde se observa aorta cabalgando el septo pero con flujo hacia aorta redireccionado por la presencia de parche en región perimembranosa, tabique sin shunt residual, FEVI $70 \%$, válvulas normales, insuficiencia tricuspídea mínima, con presión sistólica de arteria pulmonar de $15 \mathrm{mmHg}$, arco aórtico volteado para la izquierda sin alteraciones en trayecto, no hay persistencia de conducto arterioso.

La paciente evoluciona favorablemente, se mantiene hospitalizada en UCI bajo ventilación mecánica, recibe antibioticoterapia, vasoactivos, analgesia y sedación, luego de tres días en cuidados intensivos es dada de alta a piso de hospitalización general donde permanece 10 días y es dada de alta a domicilio con tratamiento farmacológico a base de furosemida a dosis de $3.5 \mathrm{mg}$ y aspirina $81 \mathrm{mg}$ una vez al día durante 3 meses y en buenas condiciones generales.

\section{DISCUSIÓN}

Un diagnóstico de Tetralogía de Fallot se puede confirmar mediante una ecocardiografía fetal o una ecocardiografía postnatal [8]. Sin embargo, aunque la ecocardiografía puede revelar la anatomía en muchos pacientes con Tetralogía de Fallot, en ocasiones el cateterismo cardíaco es necesario para definir aún más la anatomía [4]. El diagnóstico en el periodo neonatal, de igual manera se puede sospechar mediante la presentación de cianosis y soplo sistólico para esternal izquierdo asociado a una radiografía de tórax característica con "silueta cardiaca en forma de zapato zueco" [9] como el que presentaba la paciente a los 6 meses.

El ecocardiograma transesofágico intraoperatorio también ha tomado un papel importante en lo que al tratamiento respecta debido a que provee información detallada de la anatomía, la función cardíaca, y la corrección quirúrgica, además ya que aporta datos clave para que el quipo cardiovascular, llevando a un procedimiento quirúrgico de mejores resultados [10].

El tratamiento de la Tetralogía de Fallot siempre es quirúrgico, la mayoría de los pacientes se someten a una corrección total como su intervención inicial antes de un año de edad, las derivaciones pueden ser necesarias debido a episodios hipercianóticos refractarios médicamente o una obstrucción grave del tracto de salida del ventrículo derecho en lactantes que inicialmente no son candidatos aceptables para reparación intracardiaca debido a prematuridad, arterias pulmonares hipoplásicas o anatomía de arteria coronaria, además permiten el aplazamiento de la reparación electiva completa al proporcionar un flujo sanguíneo pulmonar estable requerido para la supervivencia [5] entre las derivaciones paliativas tenemos la creación de una fistula sistémico pulmonar (Blalock-Taussig) donde se coloca un injerto sintético desde la arteria innominada o subclavia hasta la arteria pulmonar ipsilateral.

El seguimiento se realiza anualmente con exámenes de control para evidenciar el progreso del cuadro, pero además se puede realizar cardio-RM en el seguimiento de la estenosis o insuficiencia pulmonar, las ramas pulmonares, la aorta ascendente y la función VD (dilatación, disfunción y/o fibrosis), siendo la fibrosis ventricular diagnosticada con cardio-RM mediante realce tardío y localizado en la región de ampliación transanular del tracto de salida VD en este grupo de pacientes lo que ha demostrado ser un marcador de eventos clínicos adversos, sin embargo no se dispone al momento en nuestro sistema de salud, por lo que se utiliza preferiblemente ecocardiograma en sus diferentes modalidades [11].

En un estudio realizado en México en el año del 2015 por Galicia et al, con seguimiento a 6 años, la paliación con fístula Blalock-Taussig modificada se realizó en 5 de los casos, evidenciando crecimiento y no distorsión de las ramas, funcionalidad del $90 \%$ en el momento de la corrección total, con fallo de la fístula (un caso), sin influir en los resultados de la cirugía posterior [12].

En nuestro caso pese a tener una complicación en un inicio como fue la hiperfunción de fistula sistémico pulmonar izquierda con hipercongestión unilateral pulmonar derecha [13], que se resolvió posteriormente, se observó a los 5 años una fistula sistémico pulmonar funcionante, pero insuficiente, siendo la paciente candidata para la corrección quirúrgica total.

La reparación completa de la tetralogía de Fallot consiste en el cierre de la comunicación interventricular con un parche, la ampliación del tracto de salida del ventrículo derecho con resección muscular y valvuloplastia pulmonar, y un parche limitado a 
través del anillo pulmonar o el tronco pulmonar si es necesario. Esta se ha realizado exitosamente en las últimas décadas, con mortalidad inferior al $5 \%$ con mejoras sustanciales en la hemodinámica [14, 15]; sin embargo, existen efectos adversos tardíos como insuficiencia progresiva ventricular derecha, arritmias y muerte súbita [12] que deben ser considerados y explicados al paciente y sus familiares.

\section{CONCLUSIONES}

La cirugía de corrección total de Tetralogía de Fallot luego de procedimientos paliativos previos, con control por ecocardiograma transesofágico transoperatorio ofrece mejores oportunidades de éxito en el acto operatorio, permitiendo una evolución favorable del paciente con una notable mejoría en su calidad de vida.

\section{RECOMENDACIONES}

Gracias al avance en las estrategias medico quirúrgicas, y con el adecuado seguimiento de los pacientes ha disminuido la morbilidad y mortalidad en estos casos, estimando que ahora el $89 \%$ de los enfermos logra llegar a la vida adulta.

A pesar de la sobrevida a largo plazo, y de la excelente calidad de vida que se obtiene en estos pacientes con la corrección total de la tetralogía de Fallot, la insuficiencia progresiva del ventrículo derecho ocurre, por lo que ninguno de estos pacientes debe considerarse curado, debiendo tenerse un seguimiento continuo, y solo así se podrá elegir el momento óptimo para el implante o corrección posterior de la válvula pulmonar.

Establecer un diagnóstico temprano y realizar estudios complementarios es un pilar fundamental para la posterior valoración y decisión del tipo de cirugía a requerir ya sea paliativa o correctiva, además de la experiencia del equipo quirúrgico según sea cada caso, para lograr mejorar la calidad de vida de estos pacientes, pero además de llegar a corregir los diferentes defectos presentes en esta patología la meta del tratamiento quirúrgico debe incluir: la prevención de las complicaciones a largo plazo y disminuir la probabilidad de re operaciones tempranas y tardías, permitiendo una calidad de vida satisfactoria en nuestros pacientes.
Actualmente las guías recomiendan la conformación de Heart Team a nivel de varias instituciones como se evidencia en este caso permitió valorar el momento adecuado para realizar tanto cirugía paliativa y luego correctiva, cabe mencionar que el aumento de los métodos de diagnóstico permite elegir el tratamiento y el tiempo oportuno, además de la experiencia del grupo médico la incorporación de nuevas subespecialidades a nivel local permiten obtener resultados locales comparables a centros de alta experiencia a nivel nacional y mundial.

\section{ASPECTOS BIOETICOS}

El presente caso clínico se realizó bajo el consentimiento informado del paciente, con total confidencialidad de datos personales.

\section{INFORMACIÓN DE LOS AUTORES}

- López Rodríguez Javier Arturo. Especialista en Medicina (Cirugía Cardiotorácica). Clínica Santa Inés. Cardiología. Cirugía Cardiotorácica. Cuenca- Azuay- Ecuador.

e-mail: javier.lopez@ucuenca.edu.ec

ORCID: https://orcid.org/0000-0002-7762-0238

- Carchi Heras Mayra Alexandra. Médico General. Clínica Santa Inés. Cardiología. Cirugía Cardiotorácica. Cuenca- Azuay- Ecuador e-mail: ale.carchi@outlook.com

ORCID: https://orcid.org/0000-0001-6906-617X

\section{CONTRIBUCIÓN DE LOS AUTORES}

Los autores declaran haber contribuido de manera similar en la concepción, diseño, análisis e interpretación de los datos, redacción del manuscrito y aprobación de la versión final, así como estar en capacidad de responder de todos los aspectos del mismo.

\section{CONFLICTO DE INTERESES}

Los autores no reportan conflicto de intereses

\section{FUENTES DE FINANCIAMIENTO}

\section{Autofinanciado}




\section{REFERENCIAS BIBLIOGRAFICAS}

1. Doyle $\mathrm{T}, \mathrm{Kavanaugh-McHugh} \mathrm{A}$. Pathophysiology, clinical features, and diagnosis of tetralogy of Fallot. UpToDate 2018 Disponible en: https://www.uptodate. com/contents/pathophysiology-clinicalfeatures-and-diagnosis-of-tetralogy-of-fallot

2. Vanegas E, Correa J, Núñez F. Tetralogía de Fallot. En Díaz G. Cardiología Pediátrica. Segunda edición. Bogotá: Editorial Distribuna. 2018. Pp 1324- 1325.

3. Telich-Taliba J. Tetralogía de Fallot: reporte de un caso y revisión de la literatura. Revista de la Facultad de Medicina de la UNAM. 2012;55(6)21-25. Disponible en: http:// www.scielo.org.mx/scielo.php?script $=$ sci arttext\&pid=S0026-17422012000600004\&In $\mathrm{g}=$ en\&nrm=iso\&tlng=es

4. MoncayoAC. Tetralogía de Fallot: diagnóstico, cirugía correctiva y manejo postoperatorio en Unidad de Cuidados Intensivos Cardiológicos Pediátricos, presentación de caso clínico y revisión bibliográfica. Revista Conocimiento Global. Cienciadigital.org 2019; 3 (1). 3553. Disponible en: http://www.cienciadigital. org/revistascienciadigital/index.php/ CienciaDigital/article/view/249

5. Friedberg MK1, Reddy S2. Right ventricular failure in congenital heart disease. Curr Opin Pediatr. 2019 Jul 25. doi: 10.1097/ MOP.0000000000000804.

6. Doyle T, Kavanaugh-McHugh A. Management and outcome of tetralogy of Fallot (internet). UpToDate: 2017. Disponible en: https://www.uptodate.com/contents/ management-and-outcome-of-tetralogy-offallot?topicRef $=5769 \&$ source=see_link

7. Roca J, Maydana A. Tetralogía de Fallot en paciente con síndrome de Down, a propósito de un caso. Rev Soc Bol Ped 2014;53(3):125128. Disponible en: http://www.scielo.org.bo/ pdf/rbp/v53n3/v53n3_a03.pdf.

8. Muñoz H. et al. Diagnóstico y manejo prenatal de patología cardiaca fetal. Rev. Med. Clin. Condes. 2016; 27(4) 447-475. Disponible en: https://www.sciencedirect.com/science/ article/pii/S0716864016300566
9. Alva-Espinosa C. Tetralogía de Fallot. Actualización del diagnóstico y tratamiento. Rev Mex Cardiol 2013; 24 (2): 87-93. Disponible en: https://www.medigraphic.com/ pdfs/cardio/h-2013/h132d.pdf

10. Orozco D, Abello M, Osorio J. Corrección de tetralogía de Fallot: papel del ecocardiograma transesofágico intraoperatorio. Revista Colombiana de Anestesiología. 2018; 40 (4). 332-334. Disponible en: https://www. sciencedirect.com/science/article/pii/ S0120334712000457

11. Gallego P, Velasco S, Aguilar R, Paré J. Actualización en técnicas de imagen cardiaca. Ecocardiografía, resonancia magnética y tomografía computarizada. Rev Esp Cardiol. 2008;61(Supl 1):109-31. Disponible en: http://www.revespcardiol.org/ es/actualizacion-tecnicas-imagen-cardiacaecocardiografia/articulo/13114647/

12. Galicia- Tornell M, Reyez- Lopez A, RuizGonzalez S, Bolio- Cerdan A, Gonzalez. Ojeda A, Fuentes- Orozco C. Tratamiento de la tetralogía de Fallot con parche transanular. Seguimiento a 6 años. Cirugía y Cirujanos. 2015; 83:478-484.

13. Hirata $Y 1$, Ono M. Surgical Management of the Patients with Tetralogy of Fallot; Longterm after Initial Repair. Kyobu Geka. 2019 Apr;72(4):258-262.

14. Sandeep B, Huang X, Xu F, Su P, Wang T, Sun $X$. Etiology of right ventricular restrictive physiology early after repair of tetralogy of Fallot in pediatric patients. J Cardiothorac Surg. 2019 May 2;14(1):84. doi: 10.1186/ s13019-019-0909-8. Disponible en https:// cardiothoracicsurgery.biomedcentral.com/ articles/10.1186/s13019-019-0909-8.

15. Guné H, Sjögren J, Carlsson M, Gustafsson R, Sjöberg P, Nozohoor S. Right ventricular remodeling after conduit replacement in patients with corrected tetralogy of Fallot evaluation by cardiac magnetic resonance. $\mathrm{J}$ Cardiothorac Surg. 2019 Apr 15;14(1):77. doi: 10.1186/s13019-019-0899-6. Disponible en: https://cardiothoracicsurgery.biomedcentral. com/articles/10.1186/s13019-019-0899-6 